\title{
Insights on the structure and stability of Licanantase: a trimeric acid-stable coiled-coil lipoprotein from Acidithiobacillus thiooxidans
}

Licanantase (Lic) is the major component of the secretome of Acidithiobacillus thiooxidans when grown in elemental sulphur. When used as an additive, Lic improves copper recovery from bioleaching processes. However, this recovery enhancement is not fully understood. In this context, our aim is to predict the 3D structure of Lic, to shed light on its structure-function relationships. Bioinformatics analyses on the amino acid sequence of Lic showed a great similarity with Lpp, an Escherichia coli Lipoprotein that can form stable trimers in solution. Lic and Lpp share the secretion motif, intracellular processing and alpha helix structure, as well as the distribution of hydrophobic residues in heptads forming a hydrophobic core, typical of coiled-coil structures. Cross-linking experiments showed the presence of Lic trimers, supporting our predictions. Taking the in vitro and in silico evidence as a whole, we propose that the most probable structure for Lic is a trimeric coiled-coil. According to this prediction, a suitable model for Lic was produced using the de novo algorithm "Rosetta Fold-and-Dock".

To assess the structural stability of our model, Molecular Dynamics (MD) and Replica Exchange MD simulations were performed using the structure of Lpp and a 14-alanine Lpp mutant as controls, at both acidic and neutral $\mathrm{pH}$. Our results suggest that Lic was the most stable structure among the studied proteins in both $\mathrm{pH}$ conditions. This increased stability can be explained by a higher number of both intermonomer hydrophobic contacts and hydrogen bonds, key elements for the stability of Lic's secondary and tertiary structure. 


\section{Fernando Abarca ${ }^{1,2}$, Sebastian E. Gutierrez-Maldonado ${ }^{1,2}$, Pilar Parada ${ }^{3}$, Patricio \\ 2 Martinez ${ }^{3}$,Alejandro Maass ${ }^{4,5}$, Tomas Perez-Acle ${ }^{1,2, *}$}

$3{ }^{1}$ Computational Biology Lab (DLab), Fundación Ciencia y Vida, Ñuñoa, Santiago, Chile

$4{ }^{2}$ Centro Interdisciplinario de Neurociencias de Valparaíso (CINV), Universidad de Valparaíso,

5 Valparaíso, Chile

$6 \quad{ }^{3}$ Biosigma S. A., Avda. Gral. San Martín 16.500, Lote 106, Colina, Santiago, Chile

$7 \quad{ }^{4}$ Mathomics, Center for Mathematical Modeling (CMM) and Center for Genome Regulation

8 (CRG), Universidad de Chile, Santiago, Chile

$9 \quad{ }^{5}$ Department of Mathematical Engineering, Universidad de Chile, Santiago, Chile

10 *Correspondence to: Tomas Perez-Acle, Ph.D., Computational Biology Lab (DLab), Fundación

11 Ciencia y Vida, 7780272 Santiago, Chile. E-mail: tomas@dlab.cl 
Introduction

13 Bioleaching is a process with increasing interest for the mining industry. It consists in the release

14 of heavy metals from insoluble ores through biological oxidation. Its importance relies in its low cost compared to flotation, smelting and conversion technologies that make the treatment of lowgrade minerals feasible, thereby increasing the ore reserves available for exploitation. Moreover,

17 bioleaching has less environmental impact than the traditional leaching process (Rawlings 2004).

18 Recently, Bobadilla Fazzini et al. (Bobadilla Fazzini et al. 2011) studied the secretome of gram-

19 negative bioleaching bacteria Acidithiobacillus thiooxidans and Acidithiobacillus ferrooxidans, when grown in the presence of elemental sulphur. The major protein component of the secretome

21 of Acidithiobacillus thiooxidans was a lipoprotein termed as Licanantase (Lic). Bioleaching assays showed that the addition of secretome fractions enriched in Lic, resulted in an enhanced copper recovery from chalcopyrite (Bobadilla Fazzini et al. 2011). The authors suggested a

24 possible participation of Lic as a surfactant agent, by removing the hydrophobic barrier formed by elemental sulphur over the surface of ores during chalcopyrite bioleaching. However, the mechanism by which the bioleaching rate is enhanced is not yet fully understood. Despite the authors reported the amino acid sequence of Lic, the lack of structural information impeded

28 further insights about its function. Within this context, our interest is to predict the structure of

29 Lic in order to get insights on its structure-function relationships that may give us a glimpse on

30 the role of this protein during bioleaching. To do so, we performed several bioinformatics

31 analyses using the amino acid sequence of Lic. We found that, despite a low sequence identity,

32 Lic shows a great similarity with a Lipoprotein (Lpp) of Escherichia coli and with its engineered

33 mutants that contain larger alanine-zipper domains (Liu et al. 2002; Liu et al. 2003; Liu \& Lu

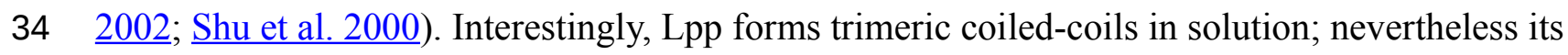

35 poly-alanine mutants lose stability, as their alanine zipper domains are larger. Based on this

36 evidence, we propose that the most probable structure for Lic is a trimeric coiled-coil. To further 
37 explore our structural hypothesis, we used de novo modelling to predict the 3D structure of Lic

38 and Replica Exchange Molecular Dynamics (REMD) to assess its pH stability, using as controls

39 the 3D structures of Lpp and a 14-alanine Lpp mutant (Ala-14). As a whole, our findings suggest

40 that Lic exhibits sequence and structural features that improve its stability at the extreme low-pH

41 environment where it performs its function. These features could be relevant to produce

42 engineered versions of this protein in order to enhance the recovery of copper by bioleaching

43 processes based on the presence of Acidithiobacillus thiooxidans.

\section{Methods}

\section{Sequence-based analyses}

46 Multiple sequence alignment was performed using ClustalW2 (oujon et al. 2010; $\underline{\text { Larkin et al. }}$

47 2007; McWilliam et al. 2013). The presence of known domains, destination signals and other

48 sequence patterns was studied using ScanProsite, and LipoP and Signal Blast for destination

49 signal (de Castro et al. 2006; Frank \& Sippl 2008; Rahman et al. 2008). TMHMM, TMPRED and

50 TOPPRED were used to detect transmembrane domains and hydrophobicity profiles (Claros \&

51 von Heijne 1994; Hofmann \& Stoffel 1993; Sonnhammer et al. 1998; von Heijne 1992).

52 Secondary structure was predicted using Jpred, NPS@, PCI-SS, Porter and SCRATCH ( $\underline{\text { Cole et }}$

53 al. 2008; Combet et al. 2000; Cheng et al. 2005; Green et al. 2009; Pollastri \& McLysaght 2005).

54 MultiCoil was used to predict coiled-coil regions (Wolf et al. 1997).

\section{Cross-linking assay}

56 E. coli cells that express recombinant Lic were treated with a hydrophobic or a hydrophilic cross-

57 linking agent, DSP and DTSSP, respectively. PBS was used as control, which was the same

58 solution used to solubilize the cross-linking agent. Proteins were analyzed by means of SDS- 
59 PAGE 15\% and Western Blot His-probe. Samples were charged in non-denaturing conditions and

60 denatured with $\beta$-mercaptoethanol.

\section{Models}

62 The Fold-and-Dock protocol, part of Rosetta 3.2 distribution, was used to predict the trimeric 3D

63 structure of Lic using a cyclic symmetry (3C) (Das et al. 2009). Fragment libraries were

64 generated by the Robetta server using the mature sequence of Lic composed of 80 amino acids

65 residues (Kim et al. 2004). 100,000 models were generated and clustered by means of a

66 clustering application implemented in the Rosetta Suite. Briefly, this program selects the 400

67 lowest energy models and, by using a RMS cutoff of $3.0 \AA$, as suggested by Shortle et al. (Shortle

68 et al. 1998), the algorithm finds the structure with the largest number of neighbors within the

69 cluster radius, creating a first cluster having this structure as the cluster center, including its

70 neighbors in the cluster. The algorithm is repeated until all structures are assigned a cluster. The

71 final model corresponds to the center structure of the highest populated cluster and it was named

72 Lic-80.

73 Lpp-56 structure [PDB: 1EQ7] was completed using MODELLER (Eswar et al. 2007) by adding

74 missing N-terminal cysteine and C-terminal lysine residues; the completed model was named

75 Lpp-58.

76 The protonation states for titratable residues of Lic-80, Lpp-58 and Ala-14 [PDB: 1JCD]

77 structures were assigned at acidic $(\mathrm{pH} 1.6)$ and neutral $(\mathrm{pH} 7.4) \mathrm{pH}$ conditions using the

78 PROPKA method (Li et al. 2005) as implemented in the PDB2PQR server (Dolinsky et al. 2007;

79 Dolinsky et al. 2004), generating two models for each protein which were used as input for MD

80 simulations. The difference between these models lies in the carboxyl groups being protonated at

81 acidic $\mathrm{pH}$, thus the modified amino acids are: D6, D15, E23, D37, D50, E64, E65, E68, E71 for 
82 Lic-80; D8, D13, D22, D27, D34, D40, D41, D50 for Lpp-58 and Ala-14; and C-terminal amino

83 acids for all three proteins.

\section{System setup for Molecular Dynamics and Replica Exchange simulations}

85 In order to produce equilibrated 3D structures that could be used to conduct further structural 86 analyses, molecular dynamics (MD) simulations were performed using the CHARMM27 force

87 field in GROMACS 4.5.4 (Bjelkmar et al. 2010; Hess et al. 2008) for each of the protonated 88 structures obtained in the previous step. To speed-up the simulation process, the OBC implicit 89 solvent approximation was used (Larsson \& Lindahl 2010; Onufriev et al. 2004). Each model 90 was minimized using the Steepest Descent algorithm with a tolerance of $100 \mathrm{~kJ} \mathrm{~mol}^{-1} \mathrm{~nm}^{-1}$. Next,

91 models were simulated for 500 ps using a harmonic restrain on alpha carbons with a constant

92 force of $10 \mathrm{~kJ} \mathrm{~mol}^{-1} \mathrm{~nm}^{-2}$. Afterwards, each simulation was run without energetic restrains for 100 93 ns. The integration step was set to 2 fs. A stochastic dynamics integrator was used with a friction 94 coefficient of $91 \mathrm{ps}^{-1}$. Coulomb and Lennard Jones interactions were handled using a cutoff of 1.3 $95 \mathrm{~nm}$. Temperature was kept constant at $303.15 \mathrm{~K}$ using velocity rescaling with a stochastic term.

96 Covalent bonds were constrained using P-Lincs algorithm (ess 2008). Trajectory frames were 97 saved every 1 ps for analyses.

98 The final structures from previous simulations were used as the starting point for Replica

99 Exchange Molecular Dynamics (REMD) simulations ( $\underline{\text { Seibert et al. 2005; }}$ Sugita \& Okamoto

100 1999). To do so, 64 replicas for each system were simulated for temperatures ranging from

$101303.15 \mathrm{~K}$ to $809.57 \mathrm{~K}(303.15,307.90,312.72,317.61,322.59,327.64,332.77,337.99,343.29$, $102348.68,354.15,359.70,365.35,371.08,376.92,382.83,388.86,394.97,401.18,407.48,413.91$, $103420.41,427.03,433.73,440.55,447.48,454.52,461.68,468.95,476.33,483.83,491.44,499.18$, $104507.03,515.01,523.11,531.35,539.71,547.96,556.59,565.34,574.24,583.28,592.47,601.81$, $105611.28,620.84,630.62,640.54,650.63,660.87,671.28,681.85,692.57,703.47,714.55,725.79$, 
106

107 calculated using the "Temperature generator for REMD-simulations" server (Patriksson \& van

108 der Spoel 2008), which was been thoroughly tested by the authors whom assure that predicted

109 and observed exchange probabilities, one of the most important factors when performing REMD

110 simulations, are correlated in $97 \%$, with minor deviations due to the different force fields tested.

111 Parameters were set for a desired exchange probability of 0.5 . Each replica was simulated for 100

112 ns, obtaining a total combined time of $6.4 \mu$ s for each system. The exchange of replicas was

113 attempted every 1 ps. Remaining parameters were the same ones used in the previous simulation

114 step.

\section{Simulation Analysis}

116 All the calculations were performed over coordinates extracted every $10 \mathrm{ps}$ from trajectories.

117 RMSD and RMSF calculations of alpha-carbons were performed over entire MD trajectories. For

118 RMSD, the first frame of each trajectory was used as reference. RMSF calculations were

119 performed for each residue, with final values corresponding to the average of the three

120 monomers.

121 Hydrogen bonds, salt bridges, hydrophobic contacts and alpha helix content were measured over

122 the last $50 \mathrm{~ns}$ of every MD trajectory and expressed as temporal averages with corresponding

123 standard deviations.

124 Hydrogen bonds were calculated using cut-offs of $3.5 \AA$ for donor-acceptor distance and $30^{\circ}$ for

125 the donor-H-acceptor angle. Salt bridges were calculated using a cut-off distance of $4.0 \AA$

126 between donor-acceptor atoms (Barlow \& Thornton 1983). Unlike hydrogen bonds, salt bridges

127 describe polar interactions that are independent of the geometry. Hydrophobic contacts were

128 calculated using a cut-off distance of $7.0 \AA$ A between centers of mass for the side chains of

129 hydrophobic residues. Only intermonomer interactions were considered for salt bridges and 
hydrophobic contacts, whereas for hydrogen bonds, intermonomer and main chain interactions

131 were considered separately. Alpha helix content was calculated by means of the STRIDE

132 program, as implemented in VMD (Frishman \& Argos 1995).

133 Unfolding temperatures $(\mathrm{Tm})$ correspond to the minimum of the first derivative with respect to

134 temperature for the observed variables measured during the last $50 \mathrm{~ns}$ of the REMD trajectories.

135 This procedure was not possible when determining Tms for intermonomer H-bonds and salt

136 bridges at neutral $\mathrm{pH}$, thus mid points were calculated instead as data did not present a sigmoidal

137 behavior (see Supporting Information Fig. S1). Calculations were performed using tools available

138 in VMD and GROMACS.

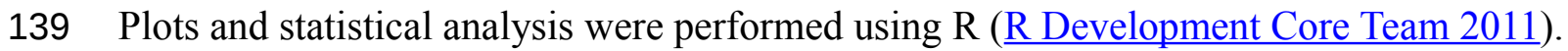

140 To reconstruct the conformational space at $303.14 \mathrm{~K}$ from the data collected at each temperature

141 during the last 50 ns of REMD simulations, the weighted histogram analysis method (WHAM)

142 was applied by using the Modular reweighting software (Sindhikara 2011). Both the number of

143 hydrophobic contacts and alpha helix content were used as the reaction coordinates for this

144 analysis.

\section{Results and discussion}

\section{Sequence analyses}

147 We searched for known domains and patterns using the amino acid sequence of Lic (Fig. 1). Our

148 findings revealed the presence of a signal peptide located in the first 21 amino acids which could

149 direct Lic to the membrane (Fig. 1, cyan box). Interestingly, a lipobox motif [ L-(A/S)-(G/A)-C]

150 (Hayashi \& Wu 1990) was detected with a potential cleavage site between residues 21|22 (Fig. 1,

151 orange and blue boxes, respectively). Prediction of potential posttranslational modifications

152 revealed that, after sorting to the inner membrane, the signal peptide could be cleaved and the

153 free Cys22 could be anchored to the membrane by its attachment to a fatty acid (Hayashi \& Wu 
154 1990; Tokuda 2009). Previous reports suggest that for lipoproteins of gram-negative bacteria, the

155 presence of an aspartate residue at position 2 (i.e., after cysteine 22) is a strong signal for the

156 retention at the inner membrane, while any other amino acids promote its translocation to the

157 outer membrane (Tokuda 2009). Lic presents an alanine at position 2, thus Lic could be

158 translocated to the outer membrane, which is in accordance to its presence in the secretome. No

159 transmembrane domains were detected other than the region already predicted as a signal peptide,

160 which is rich in hydrophobic residues (Fig. 1, red box). Secondary structure predictors coincided

161 in the existence of two segments with an alpha-helix structure: the first one corresponding to the

162 aforementioned signal peptide, whereas the second one corresponded to the processed mature

163 protein (Fig. 1, bottom scheme).

164 A FASTA (Pearson 1990) search against the PDB database detected, as the best match, a 14-

165 alanine mutant of Lpp [PDB: 1JCD] which shares a global sequence identity of $28.4 \%$ with Lic

166 given mainly by the presence of an extended patch of alanine amino acid residues. Importantly,

167 Lpp-56 [PDB: 1EQ7], which has an alanine-zipper domain composed of 3 alanines, and its

168 mutants with extended alanine-zipper domains (i.e., Ala-5, Ala-7, Ala-10 and Ala-14 [PDB:

169 1KFM, 1KFN, 1JCC and 1JCD]), were shown to form trimeric coiled-coils when crystalized (Liu

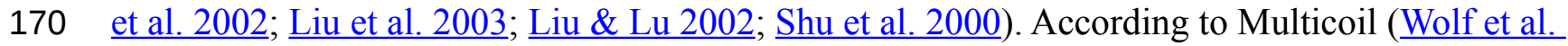

171 1997) results, the sequence of Lic shows a probability of 0.99 to form coiled-coil structures and

1720.78 to form trimeric coiled-coils in the second alpha helix segment. Notably, all predicted

173 properties for Lic (i.e., processing, secondary structure and oligomerization state) coincided with

174 those reported for Lpp (Shu et al. 2000). Considering the evidence as a whole, we propose that

175 the most probable structure for Lic is a trimeric coiled-coil. A helical-wheel diagram for the

176 proposed structure was plotted, where the hydrophobic residues can be seen aligned in positions

$177 a$ and $d$, forming a hydrophobic core with a large alanine-zipper domain (Fig. 2). 


\section{Oligomerization states}

179 In order to further evaluate our structural hypothesis, cross-linking assays were performed on Lic, 180 which was expressed heterologously in E. coli. These cells were treated with two cross-linking

181 agents: DSP, a hydrophobic reagent that can cross cell membranes, and DTSSP, a hydrophilic

182 reagent unable to cross membranes. DTSPP-treated cells showed the same band patterns than 183 DSP-treated ones (Fig. 3), suggesting that Lic can be found in the outer cell membrane, which is

184 in accordance to our bioinformatics analysis. Under native conditions, monomers, dimers and 185 trimers can be observed, while on denaturing conditions the oligomeric forms are not present.

186 The band patterns that were found (Fig. 3) resemble the patterns previously described by Choi et

187 al. for Lpp when treated with cross-linking agents (Choi et al. 1986). These results provide

188 further support for the predictions about the processing as well as the oligomeric state of Lic.

\section{Trimeric structure}

190 Knowing that Lic can form trimers, the next question to answer was if these trimers could form 191 coiled-coil structures. To evaluate the possible trimeric conformations of the mature sequence of 192 Lic, 100,000 models were generated using the Fold-and-Dock (Das et al. 2009) protocol of 193 Rosetta. As described in methods, the best 400 models were clustered, obtaining a main cluster 194 with 71 members, in which all showed a coiled-coil structure. The center structure of this cluster

195 was selected as the final model, and named as Lic-80 (Fig. 4). Root Mean-Square Fluctuation 196 (RMSF) of alpha carbons was calculated in order to measure the structural variability among the

197 members in the main cluster. The greatest variability among structures was found in their C- and 198 N-terminal ends (Fig.5). As previously proposed in Fig. 2, Lic-80 showed a hydrophobic core 199 constituted mainly of alanines, forming an alanine-zipper domain. Hydrophilic residues resulted 200 exposed to solvent and located in positions where they can form electrostatic interactions 201 between monomers (Fig. 4). 


\section{Molecular Dynamics simulations}

203 To evaluate the structural stability of Lic-80 as forming a trimeric coiled-coil structure, MD

204 simulations were performed at acidic and neutral pH. The 3D structures of Lpp-58 and Ala-14

205 were used as controls. Previous reports suggest that Lpp-58 loses structural stability in acidic pH

206 conditions due to disruption of electrostatic interactions (Bjelić et al. 2008; Dragan et al. 2004).

207 On the other hand, Ala-14 does not form stable structures in solution due to its mutations that

208 extend the alanine-zipper domain to the entire hydrophobic core, being able to form trimeric

209 coiled-coils only during the conditions applied for the crystallization process (Liu \& Lu 2002). It

210 is important to note that, due to the aminoacidic composition of these proteins, the difference

211 between MD simulations at acidic and neutral $\mathrm{pH}$ is given by the differential protonation states of

212 the carboxyl groups, specifically for the side chains of aspartate and glutamate, and the free

213 carboxyl group of the C-terminal amino acids.

214 As can be seen in Fig. 6, Root Mean-Square Deviation (RMSD) remained stable around $2 \AA$ for

215 both Lic-80 and Ala-14 and around $3 \AA$ for Lpp-58, whereas only Lpp-58 showed a higher

216 RMSD under acidic pH. RMSF profiles were similar for these three structures, showing higher

217 fluctuations in their $\mathrm{C}$ and $\mathrm{N}$-terminal ends, resembling the RMSF profile obtained for the main

218 cluster of the Rosetta models (Fig. 5). In addition, Lpp-58 showed an increase in RMSF around

219 Methionine 31 in acidic pH (Fig. 6). This phenomenon could be explained by the loss of

220 intermonomer hydrogen bonds (H-bonds) between Arginine 32 and Aspartate 27 (Fig. 7), which

221 would bring more flexibility to the trimers, thus allowing voluminous residues to accommodate

222 inside the hydrophobic core. This behavior could also explain the higher RMSD exhibited by

223 Lpp-58 at acidic pH. On the contrary, the presence of an extended alanine-zipper domain in Ala-

22414 avoids the increase in RMSD and RMSF under acidic conditions by improving the packing of

225 the coiled-coil. Our data shows this improved packing in terms of an increase in the number of 
hydrophobic contacts from 85 in Lpp-58 to 93 in Ala-14 at neutral pH and from 89 to 99 at acidic

$227 \mathrm{pH}$. Interestingly, the tightly packed coiled-coil has been previously reported by Liu \& Lu (Liu \&

$228 \mathrm{Lu} 2002)$, where they observed a decrease in the supercoil radius $\left(\mathrm{R}_{0}\right)$ for Lpp-56 from $6.1 \AA$ to

$2295.1 \AA$ in Ala-14.

230 In order to measure the effect of $\mathrm{pH}$ in nonbonding interactions, $\mathrm{pH}$-sensitive and $\mathrm{pH}$-insensitive

231 interactions were measured. Those $\mathrm{pH}$-sensitive interactions correspond to intermonomer $\mathrm{H}$ -

232 bonds and salt bridges in which protonable carboxyl groups can participate, while $\mathrm{pH}$-insensitive

233 interactions correspond to main chain H-bonds and hydrophobic contacts. As expected, the

234 number of intermonomer $\mathrm{H}$-bonds and salt bridges found at neutral $\mathrm{pH}$ was higher than the ones

235 found at acidic $\mathrm{pH}$ (Fig. 8A,B). However, the loss of electrostatic interactions due to acidic $\mathrm{pH}$

236 for Lic-80 (in average, 5 H-bonds and 6 salt bridges were broken) is less than that for Lpp-58 (in

237 average, $18 \mathrm{H}$-bonds and 9 salt bridges were broken) and Ala-14 (in average, $21 \mathrm{H}$-bonds and 9

238 salt bridges were broken), thus the effect of an acidic environment over Lic stability should be

239 lower.

240 Lic-80 showed a higher number of hydrophobic contacts than Lpp-58 and Ala-14, due to its

241 longer extension producing a larger hydrophobic interface (Fig. 8C). Also, Lic showed the

242 highest number of main chain H-bonds that stabilize the alpha helix structure. Interestingly, its

243 alpha helix content did not change under different $\mathrm{pH}$ conditions (Fig. 8D,E).

244 During these simulations Ala-14 remained folded and the effect of acidic pH on Lpp-58 stability

245 was less pronounced. These results proved the difficulty of leaving their potential energy wells

246 and highlighted the need to use methods that can improve the exploration of conformational 247 space.

248 Replica Exchange Molecular Dynamics simulations 
249 With the purpose of enhancing conformational space sampling, and being able to observe the 250 unfolding process, we performed REMD simulations for a wide range of temperatures from $251303.15 \mathrm{~K}$ to $809.57 \mathrm{~K}$. To assess thermal stability, we analyzed the reduction of nonbonding 252 interactions and alpha helix content in relation to the increase of temperature (see Supporting 253 Information Fig. S1). Thermal stability was calculated in two different ways in order to better 254 describe all the available data: the unfolding temperature (Tm) for data regarding hydrophobic 255 contacts, main chain H-bonds and helix content at both $\mathrm{pH}$ conditions; and graph midpoints for 256 intermonomer $\mathrm{H}$-bonds and salt bridges at neutral $\mathrm{pH}$. In terms of the obtained $\mathrm{Tm}$ and midpoint 257 values (Table 1), the general unfolding process can be described as follows: first, intermonomer 258 H-bonds and salt bridges are lost, followed by the loss of hydrophobic contacts, loss of the main 259 chain H-bonds and, finally, overall disruption of secondary structure (Table 1, helix content). To 260 graphically represent the unfolding process, we reconstructed the total conformational space 261 explored in REMD simulations for each structure by applying the Weighted Histogram Analysis

262 Method (WHAM) (Ferrenberg \& Swendsen 1989). For WHAM analysis, the number of

263 hydrophobic contacts and alpha helix content, accounting for trimer and monomer stability

264 respectively, were used as coordinates. The reconstructed conformational space was similar for 265 the three structures, showing two high-probability basins (Fig. 9). The first and most populated 266 basin receives contributions from folded structures at low temperatures (Fig. 9, upper-right corner 267 in each graph), while the second basin receives contributions from fully-unfolded structures at 268 high temperatures (Fig. 9, lower-left corner in each graph). Importantly, stable intermediate 269 structures were not found suggesting that the unfolding process is a one step process. All the three 270 analyzed structures showed elevated thermal stability during simulations, as high temperatures

271 were needed to induce the unfolding process. In particular, Lpp-58 showed a Tm of $539 \mathrm{~K}$ at 272 neutral $\mathrm{pH}$, versus $338 \mathrm{~K}$ which corresponds to the reported experimental value (Dragan et al. 273 2004). This could be explained due to the implicit solvent approximation that was used, which 
274 has been previously described as producing an overabundance of alpha helices in secondary

275 structures (Roe et al. 2007).

276 Dragan et al. reported that upon lowering pH conditions from neutral (7.4) to acidic (3.0), Lpp-56

277 had its Tm decrease from $338 \mathrm{~K}$ to $316 \mathrm{~K}$ (Dragan et al. 2004). However, during the performed

278 simulations, this expected decrease of Tm due to acidic $\mathrm{pH}$ was not observed. As previously

279 noted, this behavior could also be explained by the use of implicit solvent in our REMD

280 simulations. Even though the GB/SA model for implicit solvent considers both screening effects

281 and surface tension, it does not take into account the effect of hydrogen bonds formed between

282 solvent and protein, nor the effect of ions (Roe et al. 2007). Thus, we cannot rule out that the

283 observed differences in Tm for Lic-80 at acidic and neutral $\mathrm{pH}$ are accurate. However, the actual

284 difference in Tm for Lic should be smaller than that of Lpp, because its structure shows less

285 dependence on pH-sensitive hydrogen bonds and salt bridges (Fig. 8A,B). This evidence,

286 combined with that of Lic-80 showing the highest Tm among the studied structures, allows us to

287 propose the mechanisms by which Lic remains stable as a trimeric coiled-coil in the acidic

288 environments of the bioleaching media.

289 Ala-14 exhibited the smallest Tm values in both $\mathrm{pH}$ conditions. However, its thermal stability

290 was greater than expected, as Ala-14 does not form trimers and only shows a $20 \%$ of alpha helix

291 at $273 \mathrm{~K}$ (Liu \& Lu 2002). Lic-80's structure shares with Ala-14 a large alanine-zipper domain

292 that was reported as being destabilizing (Liu et al. 2002; Liu \& Lu 2002), however Lic-80

293 showed the highest Tm values. This could be explained by its greater number of $\mathrm{pH}$-insensitive

294 nonbonding interactions: 160 main chain H-bonds versus 100 in Ala-14 (Fig. 8C); and 160

295 hydrophobic contacts versus $\sim 90$ in Ala-14 (Fig. 8D). Thus, Lic-80 could be compensating the

296 weakness of these interactions by establishing a greater number of them. Unlike Ala-14, Lic-80

297 has large hydrophobic residues that could contribute stronger hydrophobic interactions,

298 specifically four methionines and one phenylalanine per monomer at the C-terminal end. 
299 Moreover, it is expected that these larger hydrophobic residues, especially methionines, could

300 improve the capability of Lic to act as a surfactant agent for elemental sulphur. This function,

301 together with the unique set of characteristics of the amino acid sequence of Lic would be key to

302 the copper recovery process during bioleaching.

\section{Conclusions}

304 In this work we showed both in silico and experimental evidence to support the notion that the

305 tridimensional structure of Lic is a trimeric coiled-coil. Although Lic exhibits a long alanine-

306 zipper domain, which has been reported as a destabilizing factor, it presented the highest

307 structural stability among the studied proteins. Thus, Lic showed a larger number of $\mathrm{pH}-$

308 insensitive nonbonding interactions that would stabilize its structure and provide resistance to

309 acid environments. Further studies are required to evaluate the participation of the phenylalanine

310 and the four methionines residues at the C-terminal end in Lic's possible function as a surfactant

311 agent. As a whole, our experimental/theoretical study has contributed to get insights on the

312 biophysical properties that allow Lic to remain stable in extreme $\mathrm{pH}$ conditions.

\section{Acknowledgments}

314 The authors would like to acknowledge Claudia Pareja for her technical work involving Fig. 1

315 and Walter Diaz for proofreading our manuscript.

\section{References}

317 Barlow DJ, and Thornton JM. 1983. Ion-pairs in proteins. Journal of molecular biology 168:867885. thermodynamic and kinetic stability of the homotrimeric coiled coil Lpp-56: A computational study. Proteins: Structure, Function, and Bioinformatics 70:810-822. Bjelkmar P, Larsson P, Cuendet MA, Hess B, and Lindahl E. 2010. Implementation of the CHARMM force field in GROMACS: Analysis of protein stability effects from correction 
338

339

340

341

342

343

344

345

346

347

348

349

350

351

352

353

354

355

356

357

358

359

360

361

362

363

364

365

366

367

368

369

370

371 maps, virtual interaction sites, and water models. Journal of Chemical Theory and Computation 6:459-466.

Bobadilla Fazzini RA, Levican G, and Parada P. 2011. Acidithiobacillus thiooxidans secretome containing a newly described lipoprotein Licanantase enhances chalcopyrite bioleaching rate. Applied microbiology and biotechnology 89:771-780.

Claros MG, and von Heijne G. 1994. TopPred II: an improved software for membrane protein structure predictions. Bioinformatics 10:685-686.

Cole C, Barber JD, and Barton GJ. 2008. The Jpred 3 secondary structure prediction server. Nucleic acids research 36:W197-201.

Combet C, Blanchet C, Geourjon C, and Deléage G. 2000. NPS@: network protein sequence analysis. Trends in biochemical sciences 25:147-150.

Cheng J, Randall aZ, Sweredoski MJ, and Baldi P. 2005. SCRATCH: a protein structure and structural feature prediction server. Nucleic acids research 33:W72-76.

Choi DS, Yamada H, Mizuno T, and Mizushima S. 1986. Trimeric structure and localization of the major lipoprotein in the cell surface of Escherichia coli. The Journal of biological chemistry 261:8953-8957.

Das R, André I, Shen Y, Wu Y, Lemak A, Bansal S, Arrowsmith CH, Szyperski T, and Baker D. 2009. Simultaneous prediction of protein folding and docking at high resolution.

Proceedings of the National Academy of Sciences of the United States of America 106:18978-18983.

de Castro E, Sigrist CJa, Gattiker A, Bulliard V, Langendijk-Genevaux PS, Gasteiger E, Bairoch A, and Hulo N. 2006. ScanProsite: detection of PROSITE signature matches and ProRule-associated functional and structural residues in proteins. Nucleic acids research 34:W362-365.

Dolinsky TJ, Czodrowski P, Li H, Nielsen JE, Jensen JH, Klebe G, and Baker Na. 2007. PDB2PQR: expanding and upgrading automated preparation of biomolecular structures for molecular simulations. Nucleic acids research 35:W522-525.

Dolinsky TJ, Nielsen JE, McCammon JA, and Baker NA. 2004. PDB2PQR: an automated pipeline for the setup of Poisson-Boltzmann electrostatics calculations. Nucleic acids research 32:W665-667.

Dragan AI, Potekhin Sa, Sivolob A, Lu M, and Privalov PL. 2004. Kinetics and thermodynamics of the unfolding and refolding of the three-stranded alpha-helical coiled coil, Lpp-56. Biochemistry 43:14891-14900.

Eswar N, Webb B, Marti-Renom MA, Madhusudhan MS, Eramian D, Shen M-Y, Pieper U, and Sali A. 2007. Comparative protein structure modeling using MODELLER. Current protocols in protein science / editorial board, John E Coligan [et al] Chapter 2:Unit 2.9Unit 2.9.

Ferrenberg A, and Swendsen R. 1989. Optimized Monte Carlo data analysis. Physical Review Letters 63:1195-1198.

Frank K, and Sippl MJ. 2008. High-performance signal peptide prediction based on sequence alignment techniques. Bioinformatics (Oxford, England) 24:2172-2176.

Frishman D, and Argos P. 1995. Knowledge-based protein secondary structure assignment. Proteins: Structure, Function, and Bioinformatics 23:566-579.

Goujon M, McWilliam H, Li W, Valentin F, Squizzato S, Paern J, and Lopez R. 2010. A new bioinformatics analysis tools framework at EMBL-EBI. Nucleic acids research 38:W695699.

Green JR, Korenberg MJ, and Aboul-magd MO. 2009. PCI-SS : MISO dynamic nonlinear protein secondary structure prediction. BMC Bioinformatics 12:1-12. 
372

373

374

375

376

377

378

379

380

381

382

383

384

385

386

387

388

389

390

391

392

393

394

395

396

397

398

399

400

401

402

403

404

405

406

407

408

409

410

411

412

413

414

415

416

417

418

419

420

Hayashi S, and Wu HC. 1990. Lipoproteins in bacteria. Journal of Bioenergetics and Biomembranes 22:451-471.

Hess B. 2008. P-LINCS: A Parallel Linear Constraint Solver for Molecular Simulation. Journal of Chemical Theory and Computation 4:116-122.

Hess B, Kutzner C, van der Spoel D, and Lindahl E. 2008. GROMACS 4: Algorithms for highly efficient, load-balanced, and scalable molecular simulation. Journal of Chemical Theory and Computation 4:435-447.

Hofmann K, and Stoffel W. 1993. TMbase - A database of membrane spanning proteins segments. Biol Chem HoppeSeyler 374:166-166.

Kim DE, Chivian D, and Baker D. 2004. Protein structure prediction and analysis using the Robetta server. Nucleic acids research 32:W526-531.

Larkin MA, Blackshields G, Brown NP, Chenna R, McGettigan PA, McWilliam H, Valentin F, Wallace IM, Wilm A, Lopez R, Thompson JD, Gibson TJ, and Higgins DG. 2007. Clustal W and Clustal X version 2.0. Bioinformatics (Oxford, England) 23:2947-2948.

Larsson P, and Lindahl E. 2010. A high-performance parallel-generalized born implementation enabled by tabulated interaction rescaling. Journal of Computational Chemistry 31:25932600.

$\mathrm{Li} \mathrm{H}$, Robertson AD, and Jensen JH. 2005. Very fast empirical prediction and rationalization of protein pKa values. Proteins 61:704-721.

Liu J, Cao W, and Lu M. 2002. Core side-chain packing and backbone conformation in Lpp-56 coiled-coil mutants. Journal of molecular biology 318:877-888.

Liu J, Dai J, and Lu M. 2003. Zinc-mediated helix capping in a triple-helical protein. Biochemistry 42:5657-5664.

Liu J, and Lu M. 2002. An alanine-zipper structure determined by long range intermolecular interactions. The Journal of biological chemistry 277:48708-48713.

McWilliam H, Li W, Uludag M, Squizzato S, Park YM, Buso N, Cowley AP, and Lopez R. 2013. Analysis Tool Web Services from the EMBL-EBI. Nucleic acids research 41:W597-600.

Onufriev A, Bashford D, and Case DA. 2004. Exploring protein native states and large-scale conformational changes with a modified generalized born model. Proteins 55:383-394.

Patriksson A, and van der Spoel D. 2008. A temperature predictor for parallel tempering simulations. Physical chemistry chemical physics : PCCP 10:2073-2077.

Pearson WR. 1990. Rapid and sensitive sequence comparison with FASTP and FASTA. Methods in enzymology 183:63-98.

Pollastri G, and McLysaght A. 2005. Porter: a new, accurate server for protein secondary structure prediction. Bioinformatics (Oxford, England) 21:1719-1720.

R Development Core Team R. 2011. R: A Language and Environment for Statistical Computing. R Foundation for Statistical Computing. p 409-409.

Rahman O, Cummings SP, Harrington DJ, and Sutcliffe IC. 2008. Methods for the bioinformatic identification of bacterial lipoproteins encoded in the genomes of Gram-positive bacteria. World Journal of Microbiology and Biotechnology 24:2377-2382.

Rawlings DE. 2004. Microbially-assisted dissolution of minerals and its use in the mining industry. Pure and Applied Chemistry 76:847-859.

Roe DR, Okur A, Wickstrom L, Hornak V, and Simmerling C. 2007. Secondary structure bias in generalized Born solvent models: comparison of conformational ensembles and free energy of solvent polarization from explicit and implicit solvation. The journal of physical chemistry B 111:1846-1857.

Seibert MM, Patriksson A, Hess B, and van der Spoel D. 2005. Reproducible polypeptide folding and structure prediction using molecular dynamics simulations. Journal of molecular biology 354:173-183.

PeerJ reviewing PDF | (v2014:05:2135:0:1:NEW 23 May 2014) 
421 Shortle D, Simons KT, and Baker D. 1998. Clustering of low-energy conformations near the

422

423

424

425

426

427

428

429

430

431

432

433

434

435

436

437

438

439 native structures of small proteins. Proceedings of the National Academy of Sciences of the United States of America 95:11158-11162.

Shu W, Liu J, Ji H, and Lu M. 2000. Core structure of the outer membrane lipoprotein from Escherichia coli at 1.9 A resolution. Journal of molecular biology 299:1101-1112.

Sindhikara DJ. 2011. Modular reweighting software for statistical mechanical analysis of biased equilibrium data. Computer Physics Communications 182:2227-2231.

Sonnhammer EL, von Heijne G, and Krogh A. 1998. A hidden Markov model for predicting transmembrane helices in protein sequences. Proceedings / International Conference on Intelligent Systems for Molecular Biology ; ISMB International Conference on Intelligent Systems for Molecular Biology 6:175-182.

Sugita Y, and Okamoto Y. 1999. Replica-exchange molecular dynamics method for protein folding. Chemical Physics Letters 314:141-151.

Tokuda H. 2009. Biogenesis of Outer Membranes in Gram-Negative Bacteria. Bioscience, Biotechnology, and Biochemistry 73:465-473.

von Heijne G. 1992. Membrane protein structure prediction. Hydrophobicity analysis and the positive-inside rule. Journal of molecular biology 225:487-494.

Wolf E, Kim PS, and Berger B. 1997. MultiCoil: a program for predicting two- and threestranded coiled coils. Protein science: a publication of the Protein Society 6:1179-1189. 


\section{Figure 1}

Multiple alignment with related sequences and sequence-based predictions for Licanantase.

Multiple alignments are color-coded to show full (black background) or partial identity (grey background) between sequences. Letters on top of each alignment correspond to helical wheel diagram positions for each residue of Lic-80 (see Fig. 2 for details). Sequence-based predictions are characterized as follows: cyan box: signal peptide sequence residues 1-22; grey box: coiled-coil prediction; red box: transmembrane region residues 4-25; orange box: lipobox motif residues 19-22; blue box: cleavage site between residues 21|22; grey lines: coil secondary structure; purple cylinder: alpha helix secondary structure.

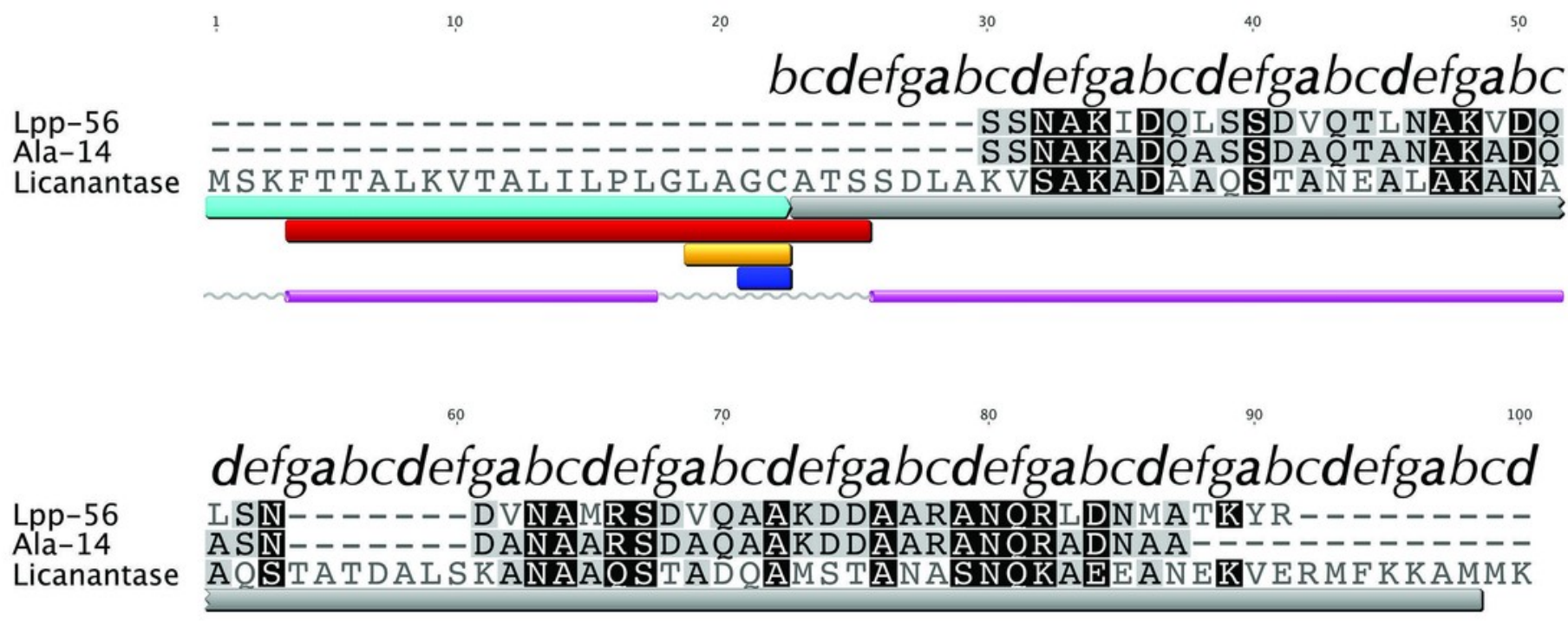




\section{Figure 2}

Helical wheel diagram of Licanantase.

Licanantase residues 22-101 can be arranged into 11 consecutive heptads where hydrophobic residues (mainly alanine) are located preferentially in positions a and $d$. Grey: Hydrophobic residues. Orange: Polar residues. Red: Acidic residues. Blue: Basic residues. Helical wheel was plotted using DrawCoil 1.0. 


\section{Figure 3}

Cross-linking assay of Licanantase in E. coli.

Cells were treated with two cross-linking agents: DSP, hydrophobic reagent that can cross cell membranes and DTSSP, hydrophilic reagent unable to cross membranes. As control, PBS solution was used. Proteins were analyzed in SDS-PAGE 15\% (A) and Western Blot His-probe (B). Samples were charged in their native state (N) and denatured (D) with a reducing agent. The un-labeled lane in both images corresponds to the molecular weight standard. Black arrows in (B), from the top, indicate the position of the trimer, dimer and monomer, respectively.

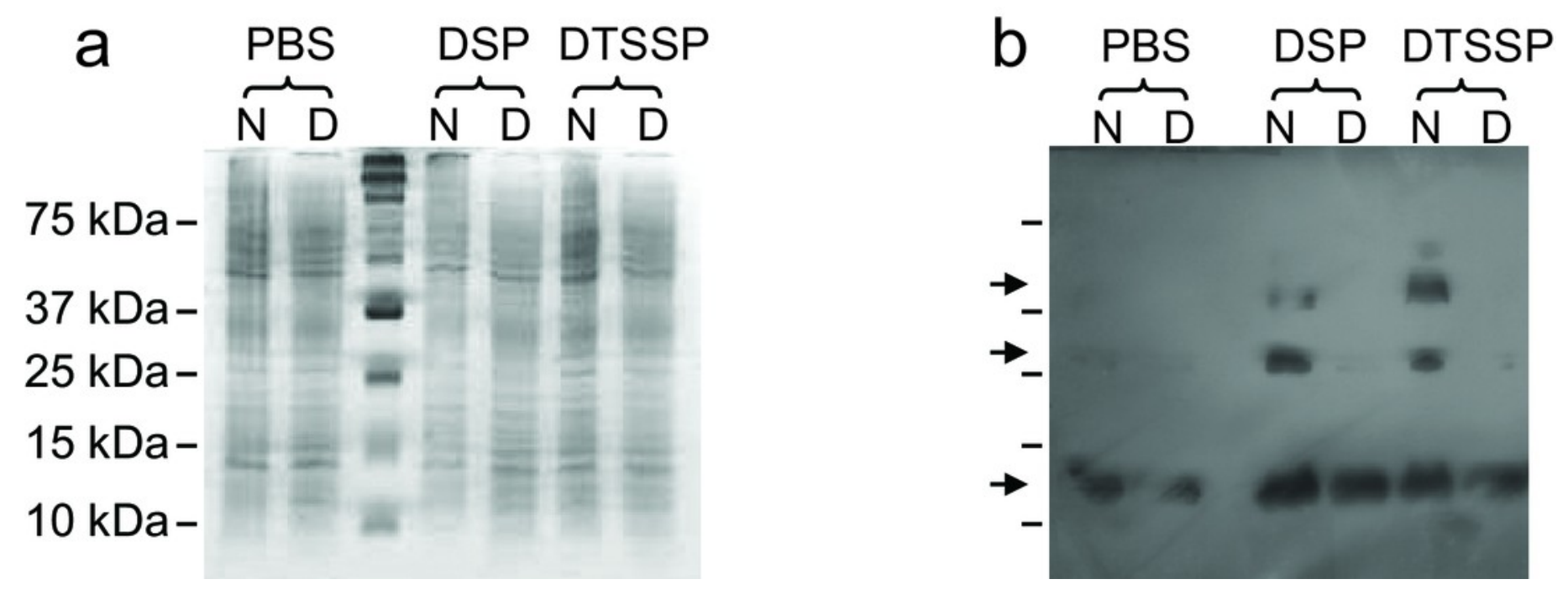




\section{Figure 4}

Licanantase model.

Trimeric Licanantase structure (Lic-80) was obtained by de novo prediction using its mature sequence of 80 aa. (A) Side view: From left to right, N-terminal to C-terminal end. (B) Top view: C-terminal end. Residue color coding: Yellow, hydrophobic. Green, polar. Blue, basic. Red, acidic.

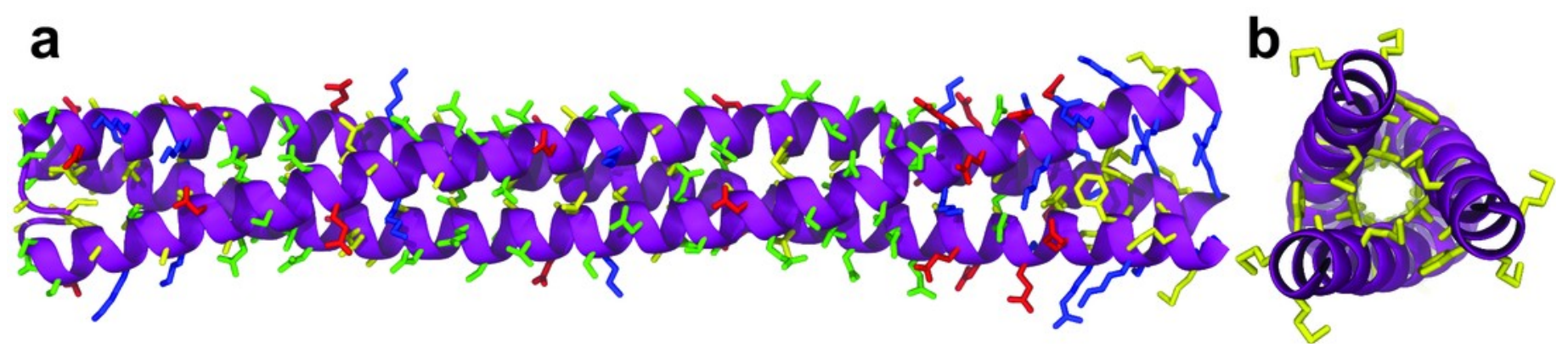




\section{Figure 5}

Root mean-square fluctuation of Licanantase models.

RMSF was calculated for alpha-carbon atoms over the 71 structures of the main cluster of Licanantase models.

Main cluster of Lic-80

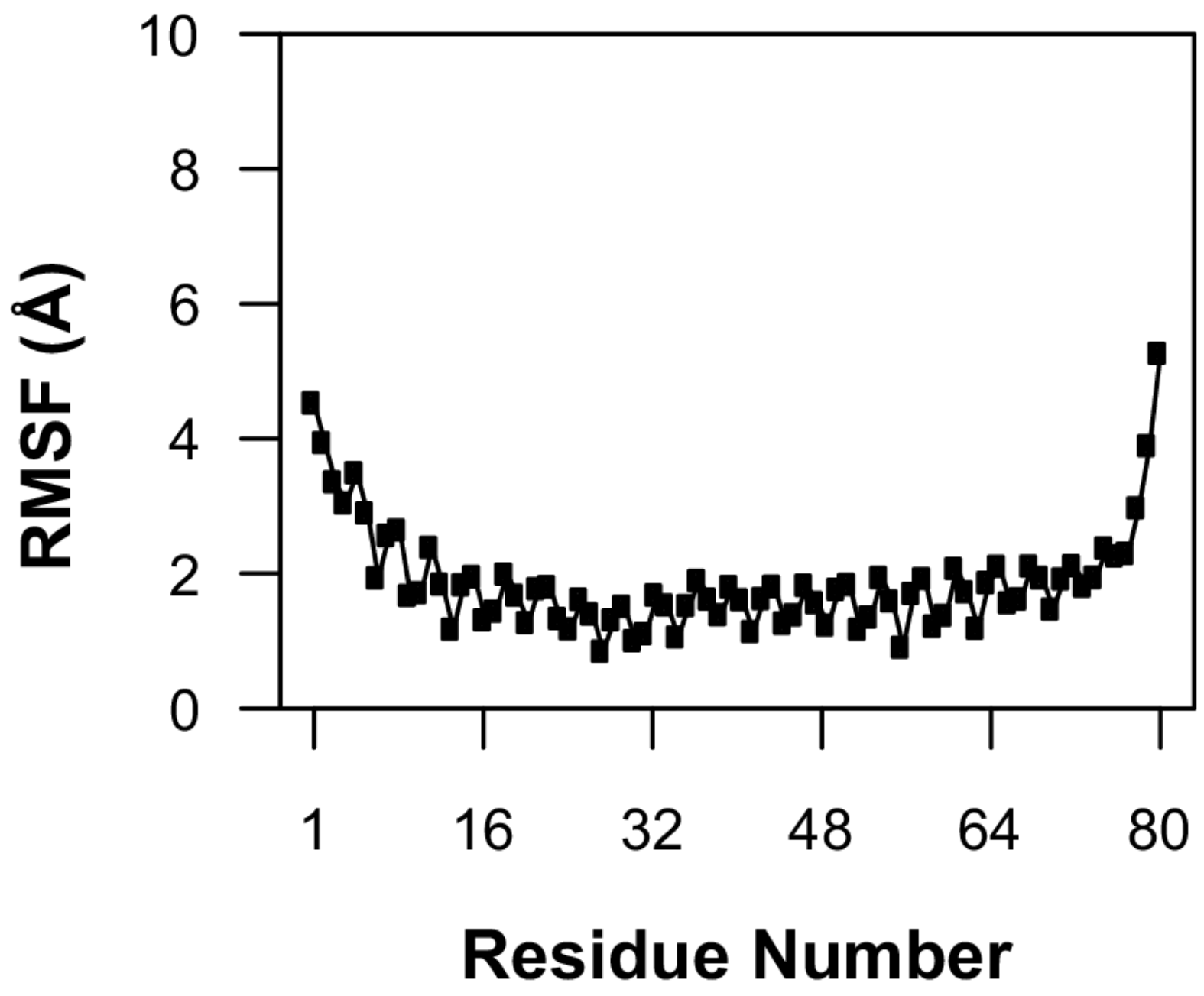




\section{Figure 6}

Root mean-square deviation and Root mean-square fluctuation of alpha carbon atoms during the entire simulations.

(A, D) Lic-80. (B, E) Lpp-58. (C, F) Ala-14. Red line: simulation at acidic pH. Blue line:

simulation at neutral $\mathrm{pH}$. Square dots and lines: average RMSF of the three monomers and its standard deviation.

a Lic-80

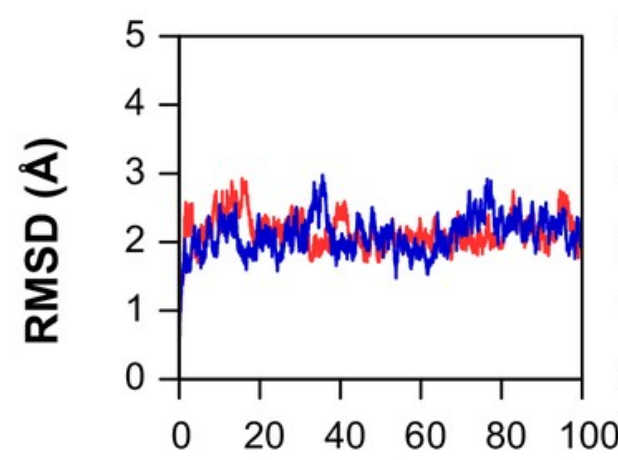

Time (ns)
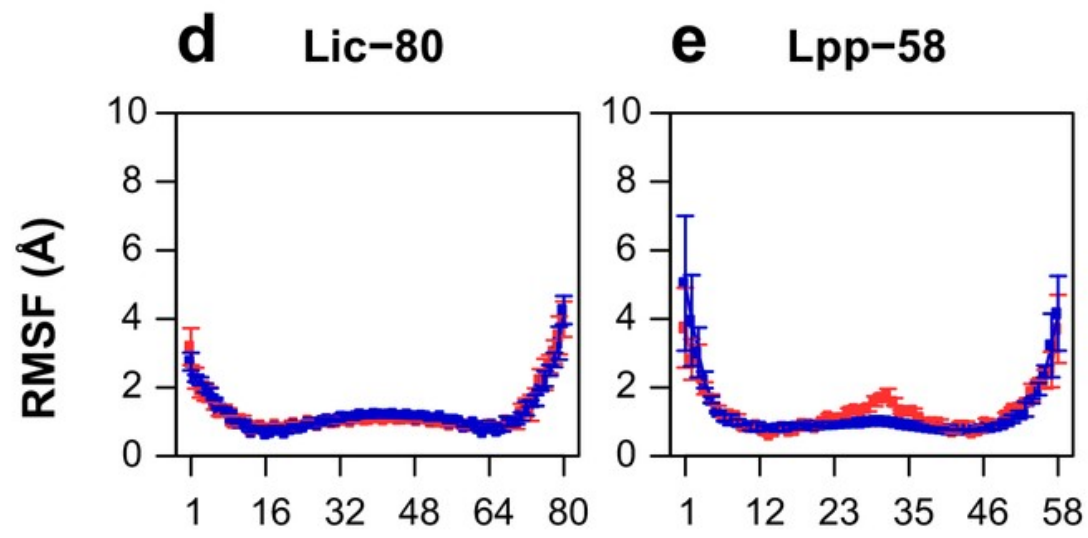
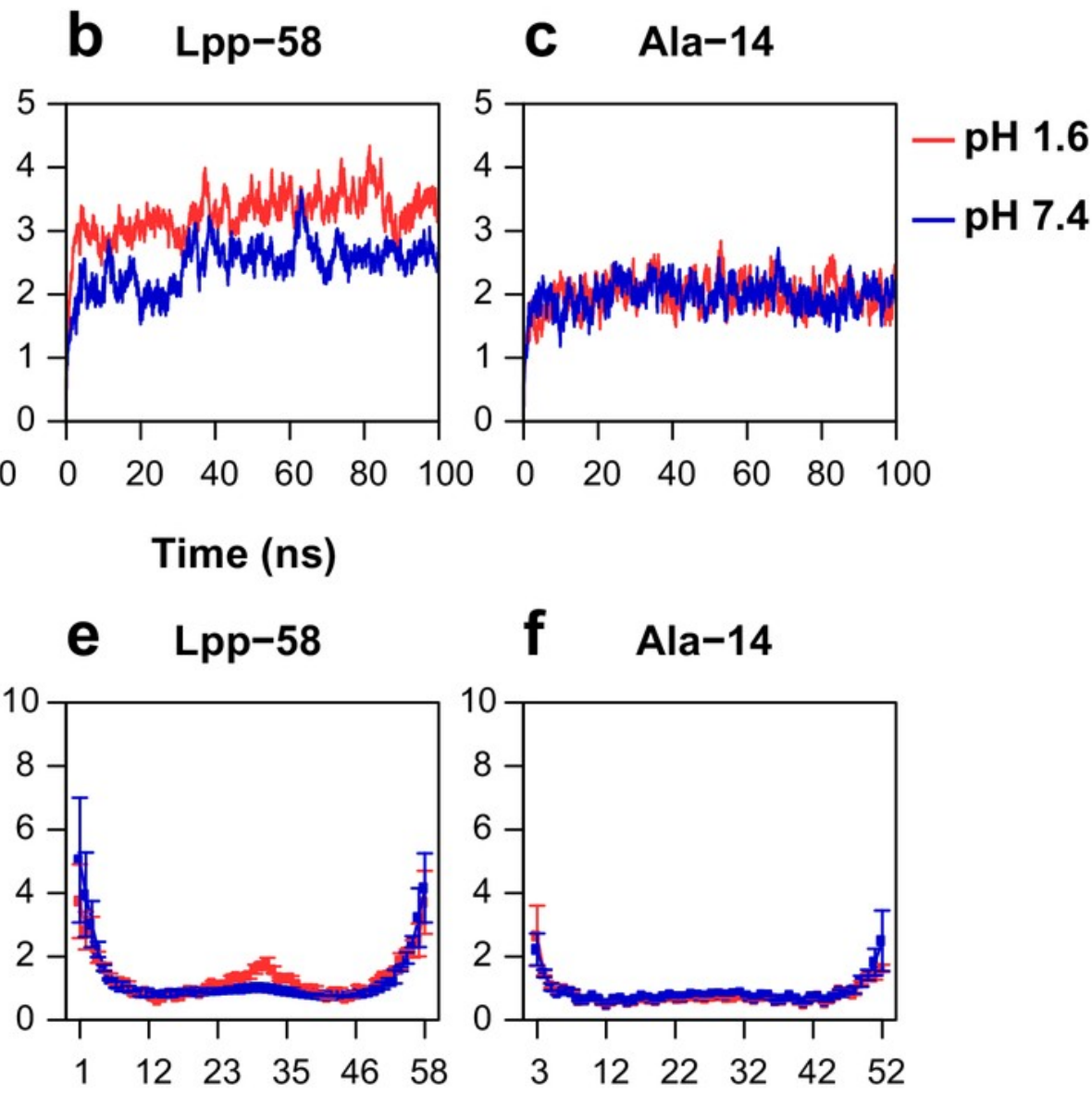

Residue Number 


\section{Figure 7}

Broken intermonomer H-bonds in Lpp-58.

Representative snapshots of Lpp-58 structure at acidic $\mathrm{pH}(\mathrm{A})$ and neutral $\mathrm{pH}(\mathrm{B})$. At acidic $\mathrm{pH}$, Arginine 32 is unable to establish intermonomer interactions with Aspartate 27 because of its protonated state, while intramonomer interactions are occasionally seen with Asparagine 29. At neutral pH, both inter- / intramonomer interactions between Arginine 32 and Asparagine 29 / Aspartate 27 can be seen. Red dotted lines: H-bonds formed between depicted residues.
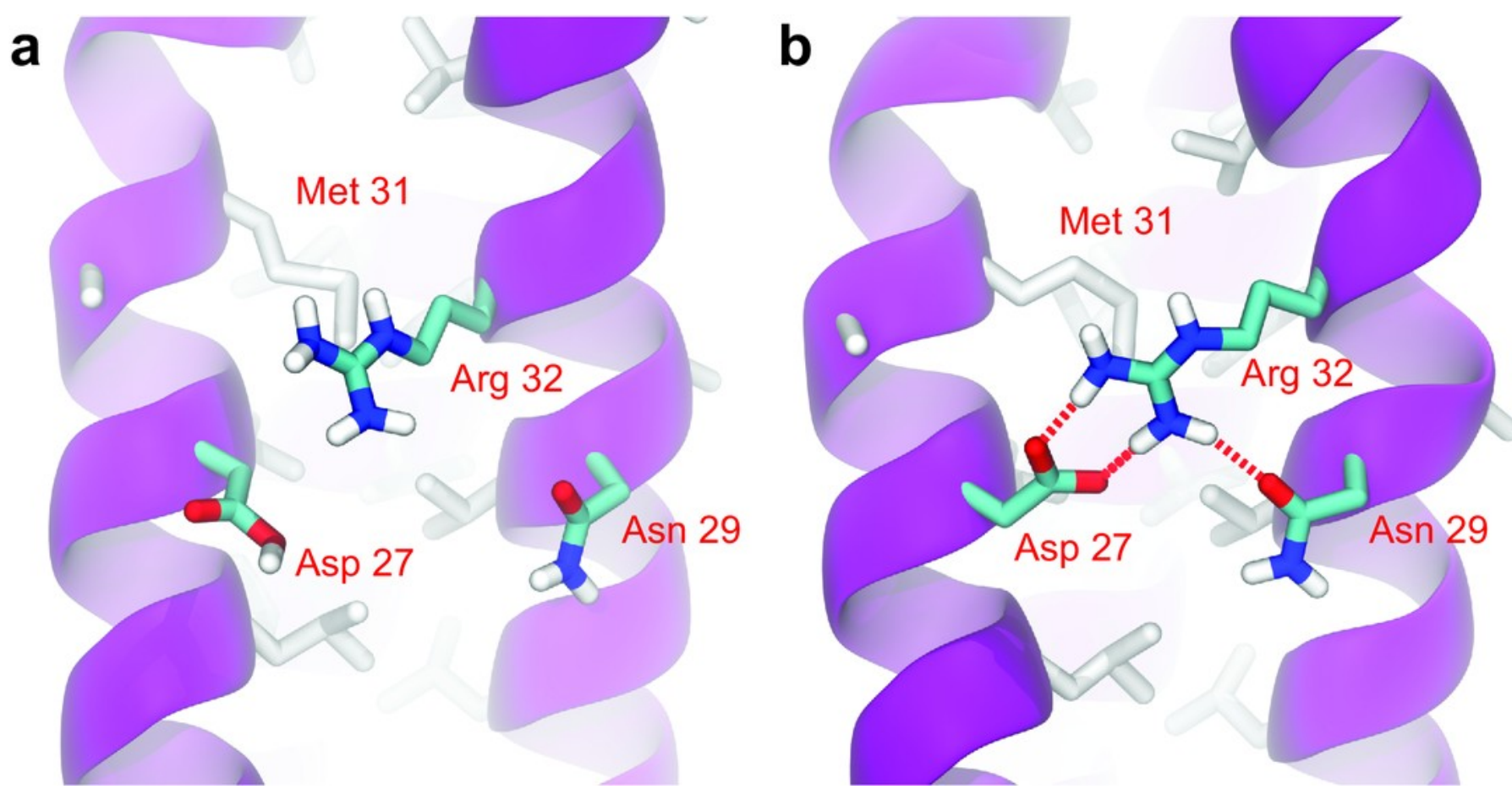


\section{Figure 8}

Property averages for the last 50 ns of simulations of Lic-80, Lpp-58 and Ala-14.

(A) Intermonomer H-bonds. (B) Intermonomer salt bridges. (C) Intermonomer hydrophobic contacts. (D) Main chain H-bonds. (E) Helix content. Bars and lines: average and its standard deviation for each property at acidic $\mathrm{pH}$ (red) and neutral $\mathrm{pH}$ (blue).

\section{a Intermonomer H-bonds b Intermonomer Salt Bridges}
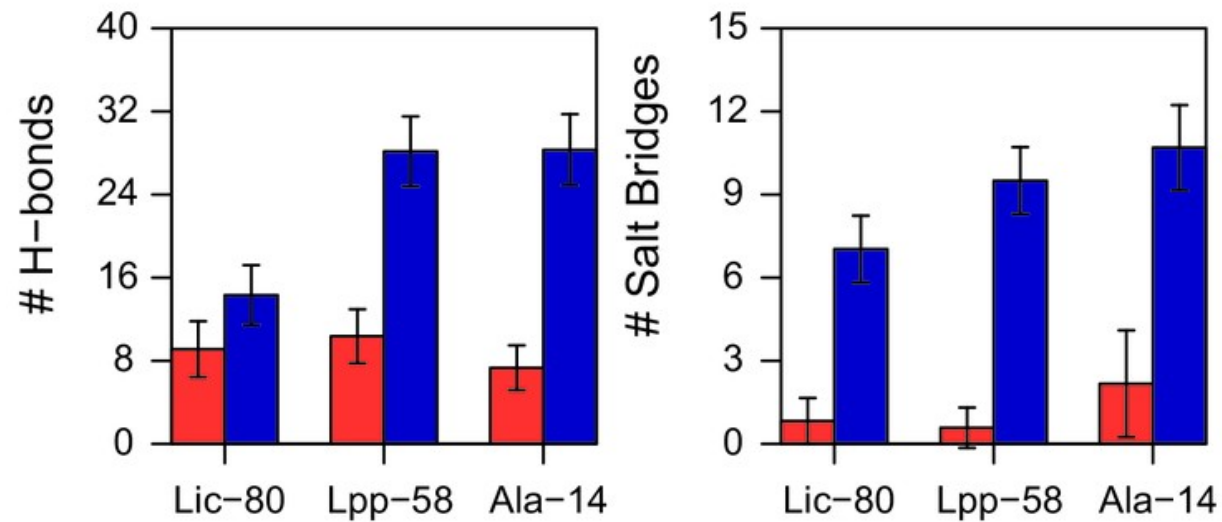

pH 1.6

pH 7.4

C Hydrophobic Contacts

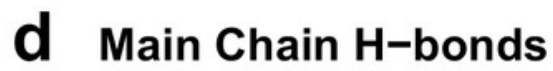

e

Helix Content
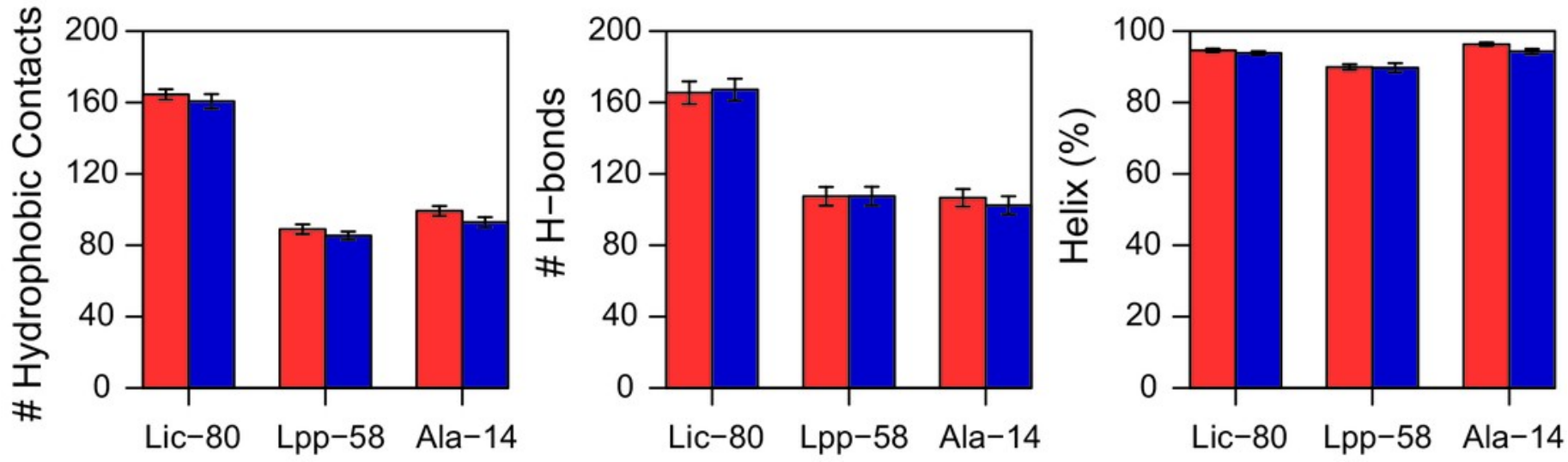


\section{Figure 9}

Representation of conformational space at $303.15 \mathrm{~K}$ obtained by WHAM for each REMD simulation.

Colored contour plot indicating the probability of finding a structure with $\mathrm{X} \%$ of helix content and $\mathrm{Y} \%$ of hydrophobic contacts at acidic $\mathrm{pH}$ (upper panels) and neutral $\mathrm{pH}$ (bottom panels) for Lic-80 (A, D), Lpp-58 (B, E) and Ala-14 (C, F).

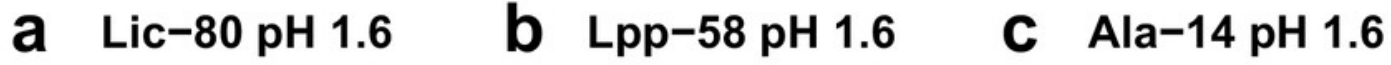

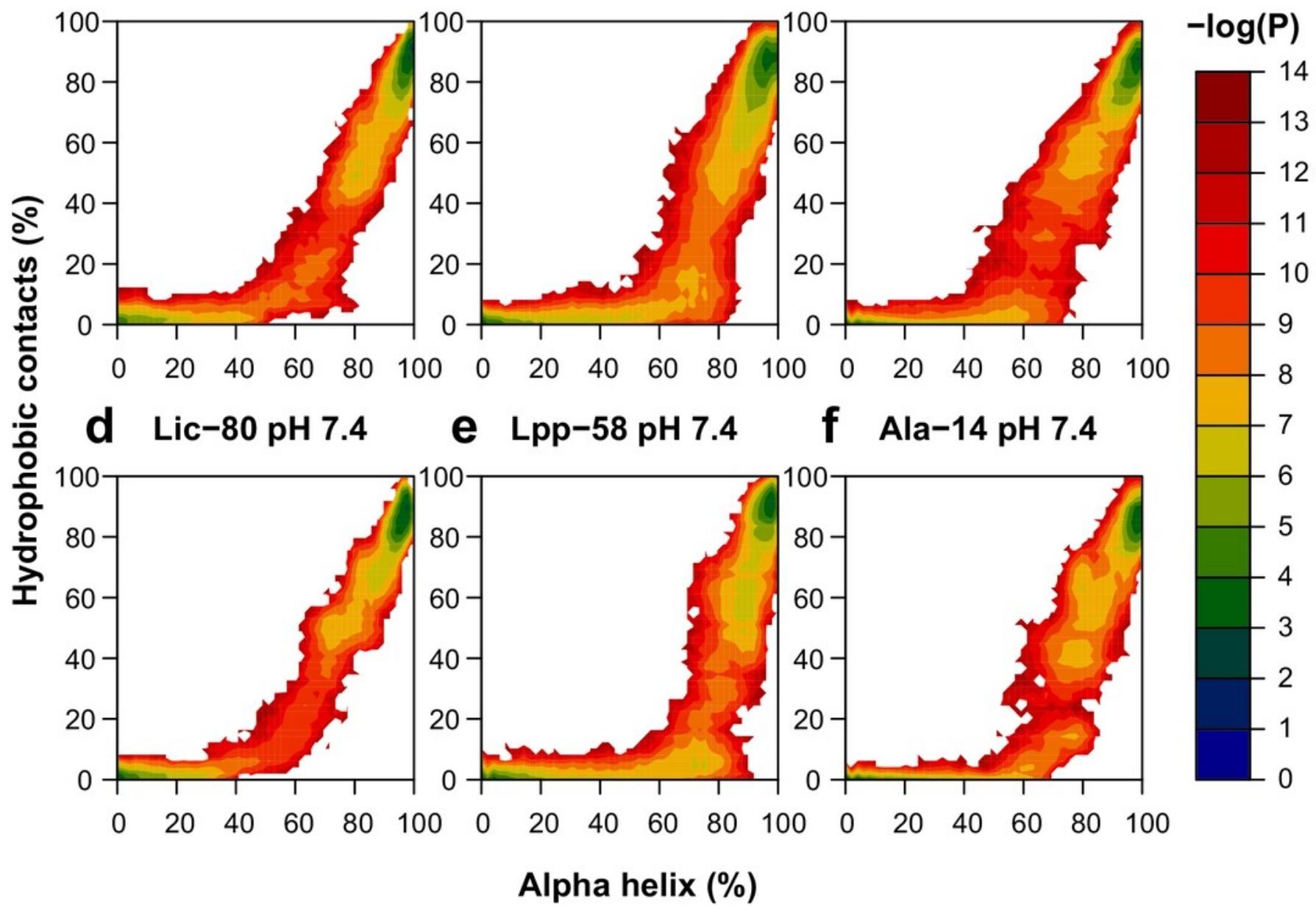




\section{Table 1 (on next page)}

Unfolding temperatures in REMD simulations 
Table 1 - Unfolding temperatures in REMD simulations

pH 7.4

\begin{tabular}{cc}
\hline \multicolumn{2}{c}{ Midpoint (K) } \\
\hline $\begin{array}{c}\text { Intermonomer } \\
\text { H-bonds }\end{array}$ & $\begin{array}{c}\text { Intermonomer } \\
\text { Salt Bridges }\end{array}$
\end{tabular}

(1)
Hydrophobic Main Chain

Contacts

Ala-14

Lpp-58

Lic-80
483.83

468.95

407.48
483.83

491.44

483.83
499.18

507.03

556.59
Main Chain
H-bonds

507.03

523.11

556.59
pH 1.6

\begin{tabular}{ccc}
\hline \multicolumn{3}{c}{$\mathbf{T m}(\mathbf{K})$} \\
\hline $\begin{array}{c}\text { Hydrophobic } \\
\text { Contacts }\end{array}$ & $\begin{array}{c}\text { Main Chain } \\
\text { H-bonds }\end{array}$ & $\begin{array}{c}\text { Helix } \\
\text { Content }\end{array}$
\end{tabular}

523.11

523.11

539.71

531.35

547.95

556.59

556.59

565.34

574.24 